\title{
Cases of integrability which correspond to the motion of a pendulum in the three-dimensional space
}

\author{
MAXIM V. SHAMOLIN \\ Lomonosov Moscow State University \\ Institute of Mechanics \\ Michurinskii Ave., 1, 119192 Moscow \\ RUSSIAN FEDERATION
}

\begin{abstract}
We systematize some results on the study of the equations of spatial motion of dynamically symmetric fi ed rigid bodies-pendulums located in a nonconservative force fields The form of these equations is taken from the dynamics of real fi ed rigid bodies placed in a homogeneous $\mathrm{fl} \mathrm{w}$ of a medium. In parallel, we study the problem of a spatial motion of a free rigid body also located in a similar force fields Herewith, this free rigid body is influence by a nonconservative tracing force; under action of this force, either the magnitude of the velocity of some characteristic point of the body remains constant, which means that the system possesses a nonintegrable servo constraint, or the center of mass of the body moves rectilinearly and uniformly; this means that there exists a nonconservative couple of forces in the system.
\end{abstract}

Key-Words: Rigid body, Pendulum, Resisting Medium, Dynamical Systems With Variable Dissipation, Integrability

Received: January 6, 2021. Revised: July 21, 2021. Accepted: August 1, 2021. Published: August 10, 2021.

\section{Model assumptions}

Let consider the homogeneous plane circle disk $\mathcal{D}$ (with the center in the point $D$ ), the plane of which perpendicular to the holder $O D$. The disk is rigidly fi ed perpendicular to the tool holder $O D$ located on the spherical hinge $O$, and it fl ws about homogeneous flui $\mathrm{fl} \mathrm{w}$. In this case, the body is a physical (spherical) pendulum. The medium $\mathrm{fl} \mathrm{w}$ moves from infinit with constant velocity $\mathbf{v}=\mathbf{v}_{\infty} \neq \mathbf{0}$. Assume that the holder does not create a resistance.

We suppose that the total force $\mathbf{S}$ of medium $\mathrm{fl} \mathrm{w}$ interaction is parallel to the holder, and point $N$ of application of this force is determined by at least the angle of attack $\alpha$, which is made by the velocity vector $\mathbf{v}_{D}$ of the point $D$ with respect to the $\mathrm{fl}$ w and the holder $O D$; the total force is also determined by the angle $\beta_{1}$, which is made in the plane of the disk $\mathcal{D}$ (thus, $\left(v, \alpha, \beta_{1}\right)$ are the spherical coordinates of the tip of the vector $\mathbf{v}_{D}$ ), and also the reduced angular velocity $\omega \cong l \Omega / v_{D}, v_{D}=\left|\mathbf{v}_{D}\right|$ ( $l$ is the length of the holder, $\Omega$ is the angular velocity of the pendulum). Such conditions arise when one uses the model of streamline fl $\mathrm{w}$ around spatial bodies $[1,2]$.

Therefore, the force $\mathbf{S}$ is directed along the normal to the disk to its side, which is opposite to the direction of the velocity $\mathbf{v}_{D}$, and passes through a certain point $N$ of the disk such that the velocity vector $\mathbf{v}_{D}$ and the force of the interaction $\mathbf{S}$ lie in the plane $O D N$ (see also $[2,3])$.

The vector $\mathbf{e}=\mathbf{O D} / l$ determines the orientation of the holder. Then $\mathbf{S}=s(\alpha) v_{D}^{2} \mathbf{e}$, where $s(\alpha)=$ $s_{1}(\alpha)$ sign $\cos \alpha$, and the resistance coefficien $s_{1} \geq 0$ depends only on the angle of attack $\alpha$. By the axesymmetry properties of the body-pendulum with respect to the point $D$, the function $s(\alpha)$ is even.

Let $D x_{1} x_{2} x_{3}=D x y z$ be the coordinate system rigidly attached to the body, herewith, the axis $D x=D x_{1}$ has a direction vector $\mathbf{e}$, and the axes $D x_{2}=D y$ and $D x_{3}=D z$ lie in the plane of the disk $\mathcal{D}$. In this case, the angle $\theta$ is made by the holder and the direction of the over-running medium $\mathrm{fl} \mathrm{w}$ (the axis $x_{0}$ ); and the angle $\psi$ is made by the projection of the holder to the immovable plane $y_{0} z_{0}$ (which perpendicular to the over-running medium $\mathrm{fl} \mathrm{w}$ ) and the axis $y_{0}$. Obviously, the angles $(\theta, \psi)=\left(\xi, \eta_{1}\right)$ are the spherical coordinates of the point $D$.

The space of positions of this spherical (physical) pendulum is the two-dimensional sphere

$$
\mathbf{S}^{2}\left\{\left(\xi, \eta_{1}\right) \in \mathbf{R}^{2}: 0 \leq \xi \leq \pi, \eta_{1} \bmod 2 \pi\right\},
$$

and its phase space is the tangent bundle of the twodimensional sphere

$$
\begin{gathered}
T_{*} \mathbf{S}^{2}\left\{\left(\dot{\xi}, \dot{\eta}_{1} ; \xi, \eta_{1}\right) \in \mathbf{R}^{4}:\right. \\
\left.0 \leq \xi \leq \pi, \eta_{1} \bmod 2 \pi\right\} .
\end{gathered}
$$


To the angular velocity, we put in correspondence $\Omega=\Omega_{1} \mathbf{e}_{1}+\Omega_{2} \mathbf{e}_{2}+\Omega_{3} \mathbf{e}_{3}\left(\mathbf{e}_{1}, \mathbf{e}_{2}, \mathbf{e}_{3}\right.$ the unit vectors of the coordinate system $\left.D x_{1} x_{2} x_{3}\right)$ the skew-symmetric matrix

$$
\tilde{\Omega}=\left(\begin{array}{ccc}
0 & -\Omega_{3} & \Omega_{2} \\
\Omega_{3} & 0 & -\Omega_{1} \\
-\Omega_{2} & \Omega_{1} & 0
\end{array}\right), \tilde{\Omega} \in \operatorname{so}(3) .
$$

The distance from the center $D$ of the disk to the center of pressure (the point $N$ ) has the form

$$
\left|\mathbf{r}_{N}\right|=r_{N}=D N\left(\alpha, \beta_{1}, \frac{l \Omega}{v_{D}}\right),
$$

where $\mathbf{r}_{N}=\left\{0, x_{2 N}, x_{3 N}\right\}=\left\{0, y_{N}, z_{N}\right\}$ in system $D x_{1} x_{2} x_{3}=D x y z$ (we omit the wave over $\Omega$ ).

We note, likely in two-dimensional case, that the model used to describe the effects of flui $\mathrm{fl} \mathrm{w}$ on fi ed pendulum is similar to the model constructed for free body and, in further, takes into account of the rotational derivative of the moment of the forces of medium influenc with respect to the pendulum angular velocity (see also $[3,4]$ ). An analysis of the problem of the spherical (physical) pendulum in a fl $\mathrm{w}$ will allow to fin the qualitative analogies in the dynamics of partially fi ed bodies and free three-dimensional ones.

\section{Set of dynamical equations in Lie algebra so $(3)$}

If $\operatorname{diag}\left\{I_{1}, I_{2}, I_{2}\right\}$ is the tensor of inertia of the bodypendulum in the coordinate system $D x_{1} x_{2} x_{3}$ then the general equation of its motion has the following form:

$$
\begin{gathered}
I_{1} \dot{\Omega_{1}}=0, I_{2} \dot{\Omega_{2}}+\left(I_{1}-I_{2}\right) \Omega_{1} \Omega_{3}= \\
=-z_{N}\left(\alpha, \beta_{1}, \frac{\Omega}{v_{D}}\right) s(\alpha) v_{D}^{2}, \\
I_{2} \dot{\Omega_{3}}+\left(I_{2}-I_{1}\right) \Omega_{1} \Omega_{2}= \\
=y_{N}\left(\alpha, \beta_{1}, \frac{\Omega}{v_{D}}\right) s(\alpha) v_{D}^{2},
\end{gathered}
$$

since the moment of the medium interaction force is determined by the following auxiliary matrix:

$$
\left(\begin{array}{ccc}
0 & x_{2 N} & x_{3 N} \\
-s(\alpha) v_{D}^{2} & 0 & 0
\end{array}\right),
$$

where $\left\{-s(\alpha) v_{D}^{2}, 0,0\right\}$ is the decomposition of the medium interaction force $\mathbf{S}$ in the coordinate system $D x_{1} x_{2} x_{3}$.

Since the dimension of the Lie algebra so(3) is equal to 3 , the system of equations (4) is a group of dynamical equations on so(3), and, simply speaking, the motion equations.
We see, that in the right-hand side of Eq. (4), firs of all, it includes the angles $\alpha, \beta_{1}$, therefore, this system of equations is not closed. In order to obtain a complete system of equations of motion of the pendulum, it is necessary to attach several sets of kinematic equations to the dynamic equation on the Lie algebra so(3).

\subsection{Cyclic first integral}

We immediately note that the system (4), by the existing dynamic symmetry

$$
I_{2}=I_{3},
$$

possesses the cyclic firs integral

$$
\Omega_{1} \equiv \Omega_{1}^{0}=\text { const. }
$$

In this case, further, we consider the dynamics of our system at zero level:

$$
\Omega_{1}^{0}=0 .
$$

Under conditions (5)-(7), the system (4) has the form of unclosed system of two equations:

$$
\begin{gathered}
I_{2} \dot{\Omega_{2}}=-z_{N}\left(\alpha, \beta_{1}, \frac{\Omega}{v_{D}}\right) s(\alpha) v_{D}^{2} \\
I_{2} \dot{\Omega_{3}}=y_{N}\left(\alpha, \beta_{1}, \frac{\Omega}{v_{D}}\right) s(\alpha) v_{D}^{2}
\end{gathered}
$$

\section{First set of kinematic equations}

In order to obtain a complete system of equations of motion, it needs the set of kinematic equations which relate the velocities of the point $D$ (i.e., the formal center of the disk $\mathcal{D}$ ) and the over-running medium fl w:

$$
\begin{gathered}
\mathbf{v}_{D}=v_{D} \cdot \mathbf{i}_{v}\left(\alpha, \beta_{1}\right)= \\
=\tilde{\Omega}\left(\begin{array}{l}
l \\
0 \\
0
\end{array}\right)+\left(-v_{\infty}\right) \mathbf{i}_{v}\left(-\xi, \eta_{1}\right),
\end{gathered}
$$

where

$$
\mathbf{i}_{v}\left(\alpha, \beta_{1}\right)=\left(\begin{array}{c}
\cos \alpha \\
\sin \alpha \cos \beta_{1} \\
\sin \alpha \sin \beta_{1}
\end{array}\right)
$$

The equation (9) expresses the theorem of addition of velocities in projections on the related coordinate system $D x_{1} x_{2} x_{3}$.

Indeed, the left-hand side of Eq. (9) is the velocity of the point $D$ of the pendulum with respect to the 
$\mathrm{fl} \mathrm{w}$ in the projections on the related with the pendulum coordinate system $D x_{1} x_{2} x_{3}$. Herewith, the vector $\mathbf{i}_{v}\left(\alpha, \beta_{1}\right)$ is the unit vector along the axis of the vector $\mathbf{v}_{D}$. The vector $\mathbf{i}_{v}\left(\alpha, \beta_{1}\right)$ has the spherical coordinates $\left(1, \alpha, \beta_{1}\right)$, which determines the decomposition (10).

The right-hand side of the Eq. (9) is the sum of the velocities of the point $D$ when you rotate the pendulum (the firs term), and the motion of the fl w (the second term). In this case, in the firs term, we have the coordinates of the vector $\mathbf{O D}=\{l, 0,0\}$ in the coordinate system $D x_{1} x_{2} x_{3}$.

We explain the second term of the right-hand side of Eq. (9) in more detail. We have in it the coordinates of the vector $\left(-\mathbf{v}_{\infty}\right)=\left\{-v_{\infty}, 0,0\right\}$ in the immovable space. In order to describe it in the projections on the related coordinate system $D x_{1} x_{2} x_{3}$, we need to make a (reverse) rotation of the pendulum at the angle $(-\xi)$ that is algebraically equivalent to multiplying the value $\left(-v_{\infty}\right)$ on the vector $\mathbf{i}_{v}\left(-\xi, \eta_{1}\right)$.

Thus, the firs set of kinematic equations (9) has the following form in our case:

$$
\begin{gathered}
v_{D} \cos \alpha=-v_{\infty} \cos \xi \\
v_{D} \sin \alpha \cos \beta_{1}=l \Omega_{3}+v_{\infty} \sin \xi \cos \eta_{1}, \\
v_{D} \sin \alpha \sin \beta_{1}=-l \Omega_{2}+v_{\infty} \sin \xi \sin \eta_{1} .
\end{gathered}
$$

The paper uses both classical methods of the qualitative theory of differential equations and methods developed by the author. The novelty and originality of the work consists in a successful combination of these two approaches.

The author understands that it would be better to include more simulations and computer experiments in this work. But, according to the author, this will be done in further articles in more detail.

Further research on this issue can be devoted to clarifying the considered model of the impact of the environment on the body, as well as improving qualitative research methods.

\section{Second set of kinematic equations}

We also need a set of kinematic equations which relate the angular velocity tensor $\widetilde{\Omega}$ and coordinates $\dot{\xi}, \dot{\eta}_{1}, \xi, \eta_{1}$ of the phase space (2) of pendulum studied, i.e., the tangent bundle $T_{*} \mathbf{S}^{2}\left\{\dot{\xi}, \dot{\eta}_{1} ; \xi, \eta_{1}\right\}$.

We draw the reasoning style allowing arbitrary dimension. The desired equations are obtained from the following two sets of relations. Since the motion of the body takes place in a Euclidean space $\mathbf{E}^{n}, n=3$ formally, at the beginning, we express the tuple consisting of a phase variables $\Omega_{2}, \Omega_{3}$, through new variable $z_{1}, z_{2}$ (from the tuple $z$ ). For this, we draw the following turn by the angle $\eta_{1}$ :

$$
\left(\begin{array}{l}
\Omega_{2} \\
\Omega_{3}
\end{array}\right)=T_{1,2}\left(\eta_{1}\right)\left(\begin{array}{l}
z_{1} \\
z_{2}
\end{array}\right),
$$

where

$$
T_{1,2}\left(\eta_{1}\right)=\left(\begin{array}{cc}
\cos \eta_{1} & -\sin \eta_{1} \\
\sin \eta_{1} & \cos \eta_{1}
\end{array}\right) .
$$

In other words, the relations

$$
\left(\begin{array}{l}
z_{1} \\
z_{2}
\end{array}\right)=T_{1,2}\left(-\eta_{1}\right)\left(\begin{array}{l}
\Omega_{2} \\
\Omega_{3}
\end{array}\right)
$$

hold, i.e.,

$$
\begin{gathered}
z_{1}=\Omega_{2} \cos \eta_{1}+\Omega_{3} \sin \eta_{2} \\
z_{2}=-\Omega_{2} \sin \eta_{1}+\Omega_{3} \cos \eta_{2} .
\end{gathered}
$$

Then we substitute the following relationship instead of the variable $z$ :

$$
z_{2}=\dot{\xi}, z_{1}=-\dot{\eta_{1}} \frac{\sin \xi}{\cos \xi} .
$$

Thus, two sets of Eqs. (12) and (13) give the second set of kinematic equations:

$$
\begin{gathered}
\Omega_{2}=-\dot{\xi} \sin \eta_{1}-\dot{\eta_{1}} \frac{\sin \xi}{\cos \xi} \cos \eta_{1}, \\
\Omega_{3}=\dot{\xi} \cos \eta_{1}-\dot{\eta_{1}} \frac{\sin \xi}{\cos \xi} \sin \eta_{1} .
\end{gathered}
$$

We see that three sets of the relations (8), (11), and (14) form the closed system of equations.

These three sets of equations include the following functions:

$$
y_{N}\left(\alpha, \beta_{1}, \frac{\Omega}{v_{D}}\right), z_{N}\left(\alpha, \beta_{1}, \frac{\Omega}{v_{D}}\right), s(\alpha) .
$$

In this case, the function $s$ is considered to be dependent only on $\alpha$, and the functions $y_{N}, z_{N}$ may depend on, along with the angles $\alpha, \beta_{1}$, generally speaking, the reduced angular velocity $\omega \cong l \Omega / v_{D}$.

\section{Problem on free body motion un- der assumption of tracing force}

Parallel to the present problem of the motion of the fi ed body, we study the spatial motion of the free axially symmetric rigid body with the frontal plane buttend (the circle disk $\mathcal{D}$ ) in the resistance force field under the quasi-stationarity conditions $[4,5]$ with the same model of medium interaction.

If $\left(v, \alpha, \beta_{1}\right)$ are the spherical coordinates of the velocity vector of the center $D$ of disk $\mathcal{D}$ lying on the 
axis of symmetry of a body, $\Omega=\left\{\Omega_{1}, \Omega_{2}, \Omega_{3}\right\}$ are the projections of its angular velocity on the axes of the coordinate system $D x_{1} x_{2} x_{3}$ related to the body (in this case, the axis of symmetry $C D$ coincides with the axis $D x_{1}=D x, C$ is the center of mass), and the axes $D x_{2}=D y$ and $D x_{3}=D z$ lie in the hyperplane of the disk; $I_{1}, I_{2}, I_{3}=I_{2}, m$ are characteristics of inertia and mass, then the dynamical part of the equations of motion in which the tangent forces of the interaction of the body with the medium are absent, has the form

$$
\begin{gathered}
\dot{v} \cos \alpha-\dot{\alpha} v \sin \alpha+\Omega_{2} v \sin \alpha \sin \beta_{1}- \\
-\Omega_{3} v \sin \alpha \cos \beta_{1}+\sigma\left(\Omega_{2}^{2}+\Omega_{3}^{2}\right)=\frac{F_{x}}{m}, \\
\dot{v} \sin \alpha \cos \beta_{1}+\dot{\alpha} v \cos \alpha \cos \beta_{1}- \\
-\dot{\beta_{1}} v \sin \alpha \sin \beta_{1}+\Omega_{3} v \cos \alpha- \\
-\Omega_{1} v \sin \alpha \sin \beta_{1}-\sigma \Omega_{1} \Omega_{2}-\sigma \dot{\Omega_{3}}=0 \\
\dot{v} \sin \alpha \sin \beta_{1}+\dot{\alpha} v \cos \alpha \sin \beta_{1}+ \\
+\dot{\beta}_{1} v \sin \alpha \cos \beta_{1}+\Omega_{1} v \sin \alpha \cos \beta_{1}- \\
-\Omega_{2} v \cos \alpha-\sigma \Omega_{1} \Omega_{3}+\sigma \dot{\Omega_{2}}=0 \\
I_{1} \dot{\Omega}_{1}=0, \\
I_{2} \dot{\Omega_{2}}+\left(I_{1}-I_{2}\right) \Omega_{1} \Omega_{3}=-z_{N}\left(\alpha, \beta_{1}, \frac{\Omega}{v}\right) s(\alpha) v^{2}, \\
I_{2} \dot{\Omega_{3}}+\left(I_{2}-I_{1}\right) \Omega_{1} \Omega_{2}=y_{N}\left(\alpha, \beta_{1}, \frac{\Omega}{v}\right) s(\alpha) v^{2},
\end{gathered}
$$

where $F_{x}=-S, S=s(\alpha) v^{2}, \sigma=C D$, in this case $\left(0, y_{N}\left(\alpha, \beta_{1}, \Omega / v\right), z_{N}\left(\alpha, \beta_{1}, \Omega / v\right)\right)$ are the coordinates of the point $N$ of application of the force $\mathbf{S}$ in the coordinate system $D x_{1} x_{2} x_{3}=D x y z$ related to the body.

The firs part of three equations of the system (15) describe the motion of the center of a mass in the three-dimensional Euclidean space $\mathbf{E}^{3}$ in the projections on the coordinate system $D x_{1} x_{2} x_{3}$. And the second part of three equation of the system (15) is obtained from the theorem on the change of the angular moment of a rigid body in the König axis.

Thus, the direct product $\mathbf{R}^{1} \times \mathbf{S}^{2} \times \operatorname{so}(3)$ of the three-dimensional manifold and the Lie algebra so(3) is the phase space of sixth-order system (15) of the dynamical equations. Herewith, since the medium influ ence force dos not depend on the position of the body in a plane, the system (15) of the dynamical equations is separated from the system of kinematic equations and may be studied independently (see also $[4,6]$ ).

\subsection{Cyclic first integral}

We immediately note that the system (15), by the existing dynamic symmetry

$$
I_{2}=I_{3},
$$

possesses the cyclic firs integral

$$
\Omega_{1} \equiv \Omega_{1}^{0}=\text { const. }
$$

In this case, further, we consider the dynamics of our system at zero level:

$$
\Omega_{1}^{0}=0 .
$$

\subsection{Nonintegrable constraint}

If we consider a more general problem on the motion of a body under the action of a certain tracing force $\mathbf{T}$ passing through the center of mass and providing the fulfillmen of the equality

$$
v \equiv \text { const }
$$

during the motion (see also $[7,8]$ ), then $F_{x}$ in system (15) must be replaced by $T-s(\alpha) v^{2}$.

As a result of an appropriate choice of the magnitude $T$ of the tracing force, we can achieve the fulfillmen of Eq. (19) during the motion. Indeed, if we formally express the value $T$ by virtue of system (15), we obtain (for $\cos \alpha \neq 0$ ):

$$
\begin{gathered}
T=T_{v}\left(\alpha, \beta_{1}, \Omega\right)=m \sigma\left(\Omega_{2}^{2}+\Omega_{3}^{2}\right)+ \\
+s(\alpha) v^{2}\left[1-\frac{m \sigma}{I_{2}} \frac{\sin \alpha}{\cos \alpha}\left[z_{N}\left(\alpha, \beta_{1}, \frac{\Omega}{v}\right) \sin \beta_{1}+\right.\right. \\
\left.\left.+y_{N}\left(\alpha, \beta_{1}, \frac{\Omega}{v}\right) \cos \beta_{1}\right]\right]
\end{gathered}
$$

This procedure can be viewed from two standpoints. First, a transformation of the system has occurred at the presence of the tracing (control) force in the system which provides the corresponding class of motions (19). Second, we can consider this procedure as a procedure that allows one to reduce the order of the system. Indeed, system (15) generates an independent fourth-order system of the following form:

$$
\begin{gathered}
\dot{\alpha} v \cos \alpha \cos \beta_{1}-\dot{\beta_{1}} v \sin \alpha \sin \beta_{1}+ \\
+\Omega_{3} v \cos \alpha-\sigma \dot{\Omega_{3}}=0 \\
\dot{\alpha} v \cos \alpha \sin \beta_{1}+\dot{\beta_{1}} v \sin \alpha \cos \beta_{1}- \\
-\Omega_{2} v \cos \alpha+\sigma \dot{\Omega}_{2}=0 \\
I_{2} \dot{\Omega_{2}}=-z_{N}\left(\alpha, \beta_{1}, \frac{\Omega}{v}\right) s(\alpha) v^{2} \\
I_{2} \dot{\Omega_{3}}=y_{N}\left(\alpha, \beta_{1}, \frac{\Omega}{v}\right) s(\alpha) v^{2}
\end{gathered}
$$


where the parameter $v$ is supplemented by the constant parameters specifie above.

The system (21) is equivalent to the system

$$
\begin{gathered}
\dot{\alpha} v \cos \alpha+ \\
+v \cos \alpha\left[\Omega_{3} \cos \beta_{1}-\Omega_{2} \sin \beta_{1}\right]+ \\
+\sigma\left[-\dot{\Omega_{3}} \cos \beta_{1}+\dot{\Omega_{2}} \sin \beta_{1}\right]=0, \\
\dot{\beta_{1}} v \sin \alpha- \\
-v \cos \alpha\left[\Omega_{2} \cos \beta_{1}+\Omega_{3} \sin \beta_{1}\right]+ \\
+\sigma\left[\dot{\Omega_{2}} \cos \beta_{1}+\dot{\Omega_{3}} \sin \beta_{1}\right]=0, \\
\dot{\Omega_{2}}=-\frac{v^{2}}{I_{2}} z_{N}\left(\alpha, \beta_{1}, \frac{\Omega}{v}\right) s(\alpha), \\
\dot{\Omega_{3}}=\frac{v^{2}}{I_{2}} y_{N}\left(\alpha, \beta_{1}, \frac{\Omega}{v}\right) s(\alpha) .
\end{gathered}
$$

We introduce new quasi-velocities in our system:

$$
\begin{gathered}
\left(\begin{array}{l}
\Omega_{2} \\
\Omega_{3}
\end{array}\right)=T_{1,2}\left(\beta_{1}\right)\left(\begin{array}{l}
z_{1} \\
z_{2}
\end{array}\right), \\
T_{1,2}\left(\beta_{1}\right)=\left(\begin{array}{cc}
\cos \beta_{1} & -\sin \beta_{1} \\
\sin \beta_{1} & \cos \beta_{1}
\end{array}\right) .
\end{gathered}
$$

In other words, the following relations

$$
\left(\begin{array}{l}
z_{1} \\
z_{2}
\end{array}\right)=T_{1,2}\left(-\beta_{1}\right)\left(\begin{array}{l}
\Omega_{2} \\
\Omega_{3}
\end{array}\right)
$$

hold, i.e.,

$$
\begin{gathered}
z_{1}=\Omega_{2} \cos \beta_{1}+\Omega_{3} \sin \beta_{2}, \\
z_{2}=-\Omega_{2} \sin \beta_{1}+\Omega_{3} \cos \beta_{2} .
\end{gathered}
$$

We can see from (22) that the system cannot be solved uniquely with respect to $\dot{\alpha}, \dot{\beta}_{1}$ on the manifold

$$
\begin{gathered}
O=\left\{\left(\alpha, \beta_{1}, \Omega_{2}, \Omega_{3}\right) \in \mathbf{R}^{4}:\right. \\
\left.\alpha=\frac{\pi}{2} k, k \in \mathbf{Z}\right\} .
\end{gathered}
$$

Thus, formally speaking, the uniqueness theorem is violated on manifold (26). Moreover, the indefinite ness occurs for even $k$ because of the degeneration of the spherical coordinates $\left(v, \alpha, \beta_{1}\right)$, and an obvious violation of the uniqueness theorem for odd $k$ occurs since the firs equation of (22) is degenerate for this case.

This implies that system (21) outside of the manifold (26) (and only outside it) is equivalent to the fol- lowing system:

$$
\begin{aligned}
& \dot{\alpha}=-z_{2}+\frac{\sigma v}{I_{2}} \frac{s(\alpha)}{\cos \alpha} \times \\
& \times {\left[z_{N}\left(\alpha, \beta_{1}, \frac{\Omega}{v}\right) \sin \beta_{1}+\right.} \\
&+\left.y_{N}\left(\alpha, \beta_{1}, \frac{\Omega}{v}\right) \cos \beta_{1}\right], \\
& \dot{z}_{2}=\frac{v^{2}}{I_{2}} s(\alpha) \times \\
& \times\left[z_{N}\left(\alpha, \beta_{1}, \frac{\Omega}{v}\right) \sin \beta_{1}+\right. \\
&\left.+y_{N}\left(\alpha, \beta_{1}, \frac{\Omega}{v}\right) \cos \beta_{1}\right]- \\
&-z_{1}^{2} \frac{\cos \alpha}{\sin \alpha}-\frac{\sigma v}{I_{2}} \frac{s(\alpha)}{\sin \alpha} z_{1} \times \\
& \times {\left[z_{N}\left(\alpha, \beta_{1}, \frac{\Omega}{v}\right) \cos \beta_{1}-\right.} \\
&\left.-y_{N}\left(\alpha, \beta_{1}, \frac{\Omega}{v}\right) \sin \beta_{1}\right], \\
& z_{1}=z_{1} z_{2} \frac{\cos \alpha}{\sin \alpha}+\left[-\frac{v^{2}}{I_{2}} s(\alpha)+\frac{\sigma v}{I_{2}} \frac{s(\alpha)}{\sin \alpha} z_{2}\right] \times \\
& \times {\left[z_{N}\left(\alpha, \beta_{1}, \frac{\Omega}{v}\right) \cos \beta_{1}-\right.} \\
&\left.-y_{N}\left(\alpha, \beta_{1}, \frac{\Omega}{v}\right) \sin \beta_{1}\right], \\
& \dot{\beta}_{1}=z_{1} \frac{\cos \alpha}{\sin \alpha}+\frac{\sigma v}{I_{2}} \frac{s(\alpha)}{\sin \alpha} \times \\
& \times {\left[z_{N}\left(\alpha, \beta_{1}, \frac{\Omega}{v}\right) \cos \beta_{1}-\right.} \\
&\left.-y_{N}\left(\alpha, \beta_{1}, \frac{\Omega}{v}\right) \sin \beta_{1}\right] .
\end{aligned}
$$

In the sequel, the dependence on the variables $\left(\alpha, \beta_{1}, \Omega / v\right)$ must be treated as the composite dependence on $\left(\alpha, \beta_{1}, z_{1} / v, z_{2} / v\right)$ by virtue of (25).

The uniqueness theorem is violated for system (22) on the manifold (26) for odd $k$ in the following sense: regular phase trajectories of system (27) pass through almost all points of the manifold (26) for odd $k$ and intersect the manifold (26) at a right angle, and also there exists a phase trajectory that completely coincides with the specifie point at all time instants. However, these trajectories are different since they correspond to different values of the tracing force.

\subsection{Constant velocity of the center of mass}

If we consider a more general problem on the motion of a body under the action of a certain tracing force $\mathbf{T}$ passing through the center of mass and providing the fulfillmen of the equality

$$
\mathbf{V}_{C} \equiv \mathbf{c o n s t}
$$

during the motion $\left(\mathbf{V}_{C}\right.$ is the velocity of the center of mass), then $F_{x}$ in system (15) must be replaced by zero since the nonconservative couple of the forces acts on the body: $T-s(\alpha) v^{2} \equiv 0$.

Obviously, we must choose the value of the tracing force $T$ as follows:

$$
T=T_{v}\left(\alpha, \beta_{1}, \Omega\right)=s(\alpha) v^{2}, \mathbf{T} \equiv-\mathbf{S} .
$$


The choice (29) of the magnitude of the tracing force $T$ is a particular case of the possibility of separation of an independent fourth-order subsystem after a certain transformation of the system (15).

Indeed, let the following condition hold for $T$ :

$$
\begin{gathered}
T=T_{v}\left(\alpha, \beta_{1}, \Omega\right)= \\
=\sum_{i, j=0, i \leq j}^{3} \tau_{i, j}\left(\alpha, \beta_{1}, \frac{\Omega}{v}\right) \Omega_{i} \Omega_{j}= \\
=T_{1}\left(\alpha, \beta_{1}, \frac{\Omega}{v}\right) v^{2}, \Omega_{0}=v .
\end{gathered}
$$

At the beginning, we introduce new quasivelocities (23)-(25).

We rewrite the system (15) for the cases (16)-(18) in the form

$$
\begin{gathered}
\dot{v}+\sigma\left(z_{1}^{2}+z_{2}^{2}\right) \cos \alpha- \\
-\sigma \frac{v^{2}}{I_{2}} s(\alpha) \sin \alpha\left[y_{N}\left(\alpha, \beta_{1}, \frac{\Omega}{v}\right) \cos \beta_{1}+\right. \\
\left.+z_{N}\left(\alpha, \beta_{1}, \frac{\Omega}{v}\right) \sin \beta_{1}\right]= \\
=\frac{T_{1}\left(\alpha, \beta_{1}, \frac{\Omega}{v}\right) v^{2}-s(\alpha) v^{2}}{m} \cos \alpha, \\
-\sigma \frac{v^{2}}{I_{2}} s(\alpha) \cos \alpha\left[y_{N}\left(\alpha, \beta_{1}, \frac{\Omega}{v}\right) \cos \beta_{1}+\right. \\
\left.z_{N}\left(\alpha, \beta_{1}, \frac{\Omega}{v}\right) \sin \beta_{1}\right]= \\
=\frac{s(\alpha) v^{2}-T_{1}\left(\alpha, \beta_{1}, \frac{\Omega}{v}\right) v^{2}}{m} \sin \alpha, \\
\dot{\Omega}_{3}=\frac{v^{2}}{I_{2}} y_{N}\left(\alpha, \beta_{1}, \frac{\Omega}{v}\right) s(\alpha), \\
\dot{\Omega}_{2}=-\frac{v^{2}}{I_{2}} z_{N}\left(\alpha, \beta_{1}, \frac{\Omega}{v}\right) s(\alpha), \\
\dot{\beta}_{1} \sin \alpha-z_{1} \cos \alpha- \\
-\frac{\sigma v}{I_{2}} s(\alpha)\left[z_{N}\left(\alpha, \beta_{1}, \frac{\Omega}{v}\right) \cos \beta_{1}-\right. \\
\left.y_{N}\left(\alpha, \beta_{1}, \frac{\Omega}{v}\right) \sin \beta_{1}\right]=0 .
\end{gathered}
$$

If we introduce the new dimensionless phase variables and the differentiation by the formulas $z_{k}=$ $n_{1} v Z_{k}, k=1,2, \quad<\cdot>=n_{1} v<^{\prime}>, n_{1}>0, n_{1}=$ const, system (31) has the following form:

$$
v^{\prime}=v \Psi\left(\alpha, \beta_{1}, Z_{1}, Z_{2}\right),
$$

$$
\begin{aligned}
& \alpha^{\prime}=-Z_{2}+\sigma n_{1}\left(Z_{1}^{2}+Z_{2}^{2}\right) \sin \alpha+ \\
& +\frac{\sigma}{I_{2} n_{1}} s(\alpha) \cos \alpha\left[y_{N}\left(\alpha, \beta_{1}, n_{1} Z\right) \cos \beta_{1}+\right. \\
& \left.+z_{N}\left(\alpha, \beta_{1}, n_{1} Z\right) \sin \beta_{1}\right]- \\
& -\frac{T_{1}\left(\alpha, \beta_{1}, n_{1} Z\right)-s(\alpha)}{m n_{1}} \sin \alpha, \\
& Z_{2}^{\prime}=\frac{s(\alpha)}{I_{2} n_{1}^{2}}\left[1-\sigma n_{1} Z_{2} \sin \alpha\right]\left[y_{N}\left(\alpha, \beta_{1}, n_{1} Z\right) \cos \beta_{1}+\right. \\
& \left.+z_{N}\left(\alpha, \beta_{1}, n_{1} Z\right) \sin \beta_{1}\right]- \\
& -Z_{1}^{2} \frac{\cos \alpha}{\sin \alpha}+\sigma n_{1} Z_{2}\left(Z_{1}^{2}+Z_{2}^{2}\right) \cos \alpha- \\
& -\frac{\sigma}{I_{2} n_{1}} Z_{1} \frac{s(\alpha)}{\sin \alpha}\left[z_{N}\left(\alpha, \beta_{1}, n_{1} Z\right) \cos \beta_{1}-\right. \\
& \left.-y_{N}\left(\alpha, \beta_{1}, n_{1} Z\right) \sin \beta_{1}\right]- \\
& -Z_{2} \frac{T_{1}\left(\alpha, \beta_{1}, n_{1} Z\right)-s(\alpha)}{m n_{1}} \cos \alpha, \\
& Z_{1}^{\prime}=\frac{1}{I_{2} n_{1}^{2}} \frac{s(\alpha)}{\sin \alpha}\left[\sigma n_{1} Z_{2} \sin \alpha-1\right] \times \\
& \times\left[z_{N}\left(\alpha, \beta_{1}, n_{1} Z\right) \cos \beta_{1}-y_{N}\left(\alpha, \beta_{1}, n_{1} Z\right) \sin \beta_{1}\right]+ \\
& +Z_{1} Z_{2} \frac{\cos \alpha}{\sin \alpha}+\sigma n_{1} Z_{1}\left(Z_{1}^{2}+Z_{2}^{2}\right) \cos \alpha- \\
& -\frac{\sigma}{I_{2} n_{1}} Z_{1} s(\alpha) \sin \alpha\left[z_{N}\left(\alpha, \beta_{1}, n_{1} Z\right) \sin \beta_{1}+\right. \\
& \left.+y_{N}\left(\alpha, \beta_{1}, n_{1} Z\right) \cos \beta_{1}\right]- \\
& -Z_{1} \frac{T_{1}\left(\alpha, \beta_{1}, n_{1} Z\right)-s(\alpha)}{m n_{1}} \cos \alpha, \\
& \beta_{1}^{\prime}=Z_{1} \frac{\cos \alpha}{\sin \alpha}+ \\
& +\frac{\sigma}{I_{2} n_{1}} \frac{s(\alpha)}{\sin \alpha}\left[z_{N}\left(\alpha, \beta_{1}, n_{1} Z\right) \cos \beta_{1}-\right. \\
& \left.-y_{N}\left(\alpha, \beta_{1}, n_{1} Z\right) \sin \beta_{1}\right], \\
& \Psi\left(\alpha, \beta_{1}, Z_{1}, Z_{2}\right)=-\sigma n_{1}\left(Z_{1}^{2}+Z_{2}^{2}\right) \cos \alpha+ \\
& +\frac{\sigma}{I_{2} n_{1}} s(\alpha) \sin \alpha\left[y_{N}\left(\alpha, \beta_{1}, n_{1} Z\right) \cos \beta_{1}+\right. \\
& \left.+z_{N}\left(\alpha, \beta_{1}, n_{1} Z\right) \sin \beta_{1}\right]+ \\
& +\frac{T_{1}\left(\alpha, \beta_{1}, n_{1} Z\right)-s(\alpha)}{m n_{1}} \cos \alpha .
\end{aligned}
$$

We see that the independent fourth-order subsystem (33)-(36) can be substituted into the fifth-orde system (32)-(36) and can be considered separately on its own four-dimensional phase space.

In particular, if condition (29) holds, then the method of separation of an independent fourth-order subsystem is also applicable. 


\section{Case where the moment of noncon- servative forces is independent of the angular velocity}

We take the function $\mathbf{r}_{N}$ as follows (the disk $\mathcal{D}$ is given by the equation $x_{1 N} \equiv 0$ ):

$$
\mathbf{r}_{N}=\left(\begin{array}{c}
0 \\
x_{2 N} \\
x_{3 N}
\end{array}\right)=R(\alpha) \mathbf{i}_{N}
$$

were

$$
\mathbf{i}_{N}=\mathbf{i}_{v}\left(\frac{\pi}{2}, \beta_{1}\right)
$$

(see (10)).

In our case

$$
\mathbf{i}_{N}=\left(\begin{array}{c}
0 \\
\cos \beta_{1} \\
\sin \beta_{1}
\end{array}\right)
$$

Thus, the equalities $x_{2 N}=R(\alpha) \cos \beta_{1}, x_{3 N}=$ $R(\alpha) \sin \beta_{1}$ hold and show that for the considered system, the moment of the nonconservative forces is independent of the angular velocity (it depends only on the angles $\alpha, \beta_{1}$ ).

And so, for the construction of the force field we use the pair of dynamical functions $R(\alpha), s(\alpha)$; the information about them is of a qualitative nature. Similarly to the choice of the Chaplygin analytical functions (see [1,2]), we take the dynamical functions $s$ and $R$ as follows:

$$
R(\alpha)=A \sin \alpha, s(\alpha)=B \cos \alpha, A, B>0 .
$$

\subsection{Reduced systems}

Theorem 1 The simultaneous equations (4), (11), (14), under conditions (5)-(7), (37), (38) can be reduced to the dynamical system on the tangent bundle (2) of the two-dimensional sphere (1).

Indeed, if we introduce the dimensionless parameter and the differentiation by the formulas

$$
b_{*}=l n_{0}, n_{0}^{2}=\frac{A B}{I_{2}},<\cdot>=n_{0} v_{\infty}<^{\prime}>,
$$

then the obtained equations have the following form $\left(b_{*}>0\right)$ :

$$
\begin{gathered}
\xi^{\prime \prime}+b_{*} \xi^{\prime} \cos \xi+\sin \xi \cos \xi-\eta_{1}^{\prime 2} \frac{\sin \xi}{\cos \xi}=0, \\
\eta_{1}^{\prime \prime}+b_{*} \eta_{1}^{\prime} \cos \xi+\xi^{\prime} \eta_{1}^{\prime} \frac{1+\cos ^{2} \xi}{\cos \xi \sin \xi}=0 .
\end{gathered}
$$

After the transition from the variables $z$ (about the variables $z$ see (13)) to the variables $w$

$$
w_{2}=-\frac{1}{n_{0} v_{\infty}} z_{2}-b_{*} \sin \xi, w_{1}=-\frac{1}{n_{0} v_{\infty}} z_{1},
$$

system (40) is equivalent to the system

$$
\left.\begin{array}{c}
\xi^{\prime}=-w_{2}-b_{*} \sin \xi, \\
w_{2}^{\prime}=\sin \xi \cos \xi-w_{1}^{2} \frac{\cos \xi}{\sin \xi}, \\
w_{1}^{\prime}=w_{1} w_{2} \frac{\cos \xi}{\sin \xi},
\end{array}\right\}
$$

on the tangent bundle

$$
\begin{gathered}
T_{*} \mathbf{S}^{2}\left\{\left(w_{2}, w_{1} ; \xi, \eta_{1}\right) \in \mathbf{R}^{4}:\right. \\
\left.0 \leq \xi \leq \pi, \eta_{1} \bmod 2 \pi\right\}
\end{gathered}
$$

of the two-dimensional sphere $\mathbf{S}^{2}\left\{\left(\xi, \eta_{1}\right) \in \mathbf{R}^{2}: 0 \leq\right.$ $\left.\xi \leq \pi, \eta_{1} \bmod 2 \pi\right\}$.

We see that the independent third-order subsystem (42) (due to cyclicity of the variable $\eta_{1}$ ) can be substituted into the fourth-order system (42), (43) and can be considered separately on its own threedimensional manifold.

\subsection{Complete list of the first integrals}

We turn now to the integration of the desired fourthorder system (42), (43) (without any simplifications i.e., in the presence of all coefficients)

First, we compare the third-order system (42) with the nonautonomous second-order system

$$
\begin{gathered}
\frac{d w_{2}}{d \xi}=\frac{\sin \xi \cos \xi-w_{1}^{2} \cos \xi / \sin \xi}{-w_{2}-b_{*} \sin \xi}, \\
\frac{d w_{1}}{d \xi}=\frac{w_{1} w_{2} \cos \xi / \sin \xi}{-w_{2}-b_{*} \sin \xi}
\end{gathered}
$$

Using the substitution $\tau=\sin \xi$, we rewrite system (45) in the algebraic form:

$$
\frac{d w_{2}}{d \tau}=\frac{\tau-w_{1}^{2} / \tau}{-w_{2}-b_{*} \tau}, \frac{d w_{1}}{d \tau}=\frac{w_{1} w_{2} / \tau}{-w_{2}-b_{*} \tau} .
$$

Further, if we introduce the uniform variables by the formulas $w_{k}=u_{k} \tau, k=1,2$, we reduce system (46) to the following form:

$$
\tau \frac{d u_{2}}{d \tau}=\frac{1-u_{1}^{2}+u_{2}^{2}-b u_{2}}{-u_{2}-b_{*}}, \tau \frac{d u_{1}}{d \tau}=\frac{2 u_{1} u_{2}-b u_{1}}{-u_{2}-b_{*}} .
$$

We compare the second-order system (47) with the nonautonomous first-orde equation

$$
\frac{d u_{2}}{d u_{1}}=\frac{1-u_{1}^{2}+u_{2}^{2}+b_{*} u_{2}}{2 u_{1} u_{2}+b_{*} u_{1}}
$$


which can be easily reduced to the exact differential equation

$$
d\left(\frac{u_{2}^{2}+u_{1}^{2}+b_{*} u_{2}+1}{u_{1}}\right)=0 .
$$

Therefore, Eq. (48) has the firs integral

$$
\frac{u_{2}^{2}+u_{1}^{2}+b_{*} u_{2}+1}{u_{1}}=C_{1}=\text { const },
$$

which in the old variables has the form

$$
\begin{gathered}
\Theta_{1}\left(w_{2}, w_{1} ; \xi\right)= \\
=\frac{w_{2}^{2}+w_{1}^{2}+b_{*} w_{2} \sin \xi+\sin ^{2} \xi}{w_{1} \sin \xi}=C_{1}= \\
=\text { const. }
\end{gathered}
$$

Remark 2 We consider system (42) with variable dissipation with zero mean (see [6, 7]), which becomes conservative for $b_{*}=0$ :

$$
\begin{gathered}
\xi^{\prime}=-w_{2}, \\
w_{2}^{\prime}=\sin \xi \cos \xi-w_{1}^{2} \frac{\cos \xi}{\sin \xi}, \\
w_{1}^{\prime}=w_{1} w_{2} \frac{\cos \xi}{\sin \xi} .
\end{gathered}
$$

It has two analytical first integrals of the form

$$
\begin{gathered}
w_{2}^{2}+w_{1}^{2}+\sin ^{2} \xi=C_{1}^{*}=\text { const }, \\
w_{1} \sin \xi=C_{2}^{*}=\text { const } .
\end{gathered}
$$

It is obvious that the ratio of the first integrals (52) and (53) is also a first integral of system (51). However, for $b_{*} \neq 0$, both functions

$$
w_{2}^{2}+w_{1}^{2}+b_{*} w_{2} \sin \xi+\sin ^{2} \xi
$$

and (53) are not first integrals of system (42), but their ratio (i.e., the ratio of the functions (54) and (53)) is a first integral of system (42) for any $b_{*}$.

Later on, we fin the obvious form of the additional firs integral of the third-order system (42). For this, at the beginning, we transform the invariant relation (49) for $u_{1} \neq 0$ as follows:

$$
\left(u_{2}+\frac{b_{*}}{2}\right)^{2}+\left(u_{1}-\frac{C_{1}}{2}\right)^{2}=\frac{b_{*}^{2}+C_{1}^{2}}{4}-1 .
$$

We see that the parameters of the given invariant relation must satisfy the condition

$$
b_{*}^{2}+C_{1}^{2}-4 \geq 0,
$$

and the phase space of system (42) is stratifie into a family of surfaces define by Eq. (55).

Thus, by virtue of relation (49), the firs equation of system (47) has the form

$$
\begin{gathered}
\tau \frac{d u_{2}}{d \tau}=\frac{2\left(1+b_{*} u_{2}+u_{2}^{2}\right)-C_{1} U_{1}\left(C_{1}, u_{2}\right)}{-u_{2}-b_{*}}, \\
U_{1}\left(C_{1}, u_{2}\right)=\frac{1}{2}\left\{C_{1} \pm \sqrt{C_{1}^{2}-4\left(u_{2}^{2}+b_{*} u_{2}+1\right)}\right\},
\end{gathered}
$$

and the integration constant $C_{1}$ is chosen from condition (56).

Therefore, the quadrature for the search of an additional firs integral of system (42) has the form

$$
\begin{gathered}
\int \frac{d \tau}{\tau}=\int \frac{\left(-b_{*}-u_{2}\right) d u_{2}}{A}, \\
A=2\left(1+b_{*} u_{2}+u_{2}^{2}\right)- \\
-C_{1}\left\{C_{1} \pm \sqrt{C_{1}^{2}-4\left(u_{2}^{2}+b_{*} u_{2}+1\right)}\right\} / 2 .
\end{gathered}
$$

Obviously, the left-hand side up to an additive constant is equal to $\ln |\sin \xi|$. If

$$
u_{2}+\frac{b_{*}}{2}=r_{1}, b_{1}^{2}=b_{*}^{2}+C_{1}^{2}-4,
$$

then the right-hand side of Eq. (57) has the form

$$
\begin{aligned}
& -\frac{1}{4} \int \frac{d\left(b_{1}^{2}-4 r_{1}^{2}\right)}{\left(b_{1}^{2}-4 r_{1}^{2}\right) \pm C_{1} \sqrt{b_{1}^{2}-4 r_{1}^{2}}}+ \\
& +b_{*} \int \frac{d r_{1}}{\left(b_{1}^{2}-4 r_{1}^{2}\right) \pm C_{1} \sqrt{b_{1}^{2}-4 r_{1}^{2}}}= \\
& =-\frac{1}{2} \ln \left|\frac{\sqrt{b_{1}^{2}-4 r_{1}^{2}}}{C_{1}} \pm 1\right| \pm \frac{b_{*}}{2} I_{1},
\end{aligned}
$$

where

$$
\begin{gathered}
I_{1}=\int \frac{d r_{3}}{\sqrt{b_{1}^{2}-r_{3}^{2}}\left(r_{3} \pm C_{1}\right)}, \\
r_{3}=\sqrt{b_{1}^{2}-4 r_{1}^{2}} .
\end{gathered}
$$

In the calculation of integral (59), the following three cases are possible.

I. $b_{*}>2$.

$$
I_{1}=-\frac{1}{2 \sqrt{b_{*}^{2}-4}} \times
$$

$$
\times \ln \left|\frac{\sqrt{b_{*}^{2}-4}+\sqrt{b_{1}^{2}-r_{3}^{2}}}{r_{3} \pm C_{1}} \pm \frac{C_{1}}{\sqrt{b_{*}^{2}-4}}\right|+
$$


$+\frac{1}{2 \sqrt{b_{*}^{2}-4}} \ln \left|\frac{\sqrt{b_{*}^{2}-4}-\sqrt{b_{1}^{2}-r_{3}^{2}}}{r_{3} \pm C_{1}} \mp \frac{C_{1}}{\sqrt{b_{*}^{2}-4}}\right|+$

+ const.

II. $b_{*}<2$.

$I_{1}=\frac{1}{\sqrt{4-b_{*}^{2}}} \arcsin \frac{ \pm C_{1} r_{3}+b_{1}^{2}}{b_{1}\left(r_{3} \pm C_{1}\right)}+$ const.

III. $b_{*}=2$.

$$
I_{1}=\mp \frac{\sqrt{b_{1}^{2}-r_{3}^{2}}}{C_{1}\left(r_{3} \pm C_{1}\right)}+\text { const. }
$$

When we return to the variable

$$
r_{1}=\frac{w_{2}}{\sin \xi}+\frac{b_{*}}{2},
$$

we obtain the fina form for the value $I_{1}$ :

I. $b_{*}>2$.

$$
\begin{gathered}
I_{1}= \\
=-\frac{1}{2 \sqrt{b_{*}^{2}-4}} \ln \left|\frac{\sqrt{b_{*}^{2}-4} \pm 2 r_{1}}{\sqrt{b_{1}^{2}-4 r_{1}^{2}} \pm C_{1}} \pm \frac{C_{1}}{\sqrt{b_{*}^{2}-4}}\right|+ \\
+\frac{1}{2 \sqrt{b_{*}^{2}-4}} \ln \left|\frac{\sqrt{b_{*}^{2}-4} \mp 2 r_{1}}{\sqrt{b_{1}^{2}-4 r_{1}^{2}} \pm C_{1}} \mp \frac{C_{1}}{\sqrt{b_{*}^{2}-4}}\right|+ \\
+ \text { const. }
\end{gathered}
$$

II. $b_{*}<2$.

$$
\begin{aligned}
& I_{1}=\frac{1}{\sqrt{4-b_{*}^{2}}} \arcsin \frac{ \pm C_{1} \sqrt{b_{1}^{2}-4 r_{1}^{2}}+b_{1}^{2}}{b_{1}\left(\sqrt{b_{1}^{2}-4 r_{1}^{2}} \pm C_{1}\right)}+ \\
&+ \text { const. }
\end{aligned}
$$

III. $b_{*}=2$.

$$
I_{1}=\mp \frac{2 r_{1}}{C_{1}\left(\sqrt{b_{1}^{2}-4 r_{1}^{2}} \pm C_{1}\right)}+\text { const. }
$$

Thus, we have found an additional firs integral for the third-order system (42), i.e., we have a complete set of firs integrals that are transcendental functions of the phase variables.

Remark 3 In the expression of the found first integral, we must formally substitute the left-hand side of the first integral (49) instead of $C_{1}$.
Then the obtained additional firs integral has the following structure similar to the transcendental firs integral from the planar dynamics):

$$
\begin{gathered}
\Theta_{2}\left(w_{2}, w_{1} ; \xi\right)= \\
=G\left(\sin \xi, \frac{w_{2}}{\sin \xi}, \frac{w_{1}}{\sin \xi}\right)=C_{2}=\text { const. }
\end{gathered}
$$

Thus, we have already found two independent firs integrals for the integration of the fourth-order system (42), (43). For its complete integrability, it suffice to fin one additional firs integral, which "attaches" Eq. (43).

Since

$$
\frac{d u_{1}}{d \tau}=\frac{u_{1}\left(2 u_{2}+b_{*}\right)}{\left(-b_{*}-u_{2}\right) \tau}, \frac{d \eta_{1}}{d \tau}=\frac{u_{1}}{\left(-b_{*}-u_{2}\right) \tau},
$$

we have

$$
\frac{d u_{1}}{d \eta_{1}}=2 u_{2}+b_{*} .
$$

Obviously, for $u_{1} \neq 0$, the following equality holds:

$$
\begin{gathered}
u_{2}=\frac{1}{2}\left(-b_{*} \pm \sqrt{b_{1}^{2}-4\left(u_{1}-\frac{C_{1}}{2}\right)^{2}}\right), \\
b_{1}^{2}=b_{*}^{2}+C_{1}^{2}-4,
\end{gathered}
$$

then integration of the quadrature

$$
\eta_{1}+\text { const }= \pm \int \frac{d u_{1}}{\sqrt{b_{1}^{2}-4\left(u_{1}-\frac{C_{1}}{2}\right)^{2}}}
$$

yields the invariant relation

$$
\begin{gathered}
2\left(\eta_{1}+C_{3}\right)= \\
= \pm \arcsin \frac{2 u_{1}-C_{1}}{\sqrt{b_{*}^{2}+C_{1}^{2}-4}}, C_{3}=\text { const. }
\end{gathered}
$$

In other words, the equality

$$
\sin \left[2\left(\eta_{1}+C_{3}\right)\right]= \pm \frac{2 u_{1}-C_{1}}{\sqrt{b_{*}^{2}+C_{1}^{2}-4}}
$$

holds and, returning to the old variables, we obtain

$$
\sin \left[2\left(\eta_{1}+C_{3}\right)\right]= \pm \frac{2 w_{1}-C_{1} \sin \xi}{\sqrt{b_{*}^{2}+C_{1}^{2}-4} \sin \xi} .
$$

In principle, in order to obtain an additional invariant relation that "attaches" Eq. (43), we could 
stop on the last equation. In this case, we must formally substitute the left-hand side of the firs integral (49) into the last expression instead of $C_{1}$.

But we perform some transformations which allow to obtain the following explicit form of the additional firs integral (in this case, we use Eq. (49)):

$$
\operatorname{tg}^{2}\left[2\left(\eta_{1}+C_{3}\right)\right]=\frac{\left(u_{1}^{2}-u_{2}^{2}-b_{*} u_{2}-1\right)^{2}}{u_{1}^{2}\left(4 u_{2}^{2}+4 b_{*} u_{2}+b_{*}^{2}\right)} .
$$

Returning to the old coordinates, we obtain an additional invariant relation of the form

$$
\begin{gathered}
\operatorname{tg}^{2}\left[2\left(\eta_{1}+C_{3}\right)\right]= \\
=\frac{\left(w_{1}^{2}-w_{2}^{2}-b_{*} w_{2} \sin \xi-\sin ^{2} \xi\right)^{2}}{w_{1}^{2}\left(4 w_{2}^{2}+4 b_{*} w_{2} \sin \xi+b_{*}^{2} \sin ^{2} \xi\right)},
\end{gathered}
$$

or, finall ,

$$
\begin{gathered}
\Theta_{3}\left(w_{2}, w_{1} ; \xi, \eta_{1}\right)= \\
=-\eta_{1} \pm \frac{1}{2} \operatorname{arctg} \frac{w_{1}^{2}-w_{2}^{2}-b_{*} w_{2} \sin \xi-\sin ^{2} \xi}{w_{1}\left(2 w_{2}+b_{*} \sin \xi\right)}= \\
=C_{3}=\text { const. }
\end{gathered}
$$

Therefore, in the considered case, the system of dynamical equations (42), (43) has three firs integrals expressing by relations (50), (67), (68), which are the transcendental functions of its phase variables (in the sense of the complex analysis) and are expressed as a finit combination of elementary functions (in this case, we use the expressions (63)-(66)).

Theorem 4 Three sets of relations (4), (11), (14) under conditions (5)-(7), (37), (38) possess three the first integrals (the complete set), which are the transcendental function (in the sense of complex analysis) and are expressed as a finite combination of elementary functions.

\subsection{Topological analogies}

Now we present two groups of analogies related to the system (15), which describes the motion of a free body in the presence of a tracking force.

The first group of analogies deals with the case of the presence the nonintegrable constraint (19) in the system. In this case the dynamical part of the motion equations under certain conditions is reduced to a system (27).

Under conditions (37), (38) the system (27) has the form

$$
\begin{gathered}
\alpha^{\prime}=-w_{2}+b \sin \alpha, \\
w_{2}^{\prime}=\sin \alpha \cos \alpha-w_{1}^{2} \frac{\cos \alpha}{\sin \alpha}, \\
w_{1}^{\prime}=w_{1} w_{2} \frac{\cos \alpha}{\sin \alpha},
\end{gathered}
$$

$$
\beta_{1}^{\prime}=w_{1} \frac{\cos \alpha}{\sin \alpha},
$$

if we introduce the dimensionless parameter, the variables, and the differentiation analogously to (39):

$$
\begin{gathered}
b=\sigma n_{0}, n_{0}^{2}=\frac{A B}{I_{2}}, z_{k}=n_{0} v w_{k}, k=1,2, \\
<\cdot>=n_{0} v<^{\prime}>
\end{gathered}
$$

Theorem 5 System (69), (70) (for the case of a free body) is equivalent to the system (42), (43) (for the case of a fixed pendulum).

Indeed, it is sufficien to substitute

$$
\xi=\alpha, \eta_{1}=\beta_{1}, b_{*}=-b .
$$

Corollary 6 1. The angle of attack $\alpha$ and the angle of sliding $\beta_{1}$ for a free body are equivalent to the angles of a body deviation $\xi=\theta$ and $\eta_{1}=\psi$, respectively, of a fixed pendulum.

2. The distance $\sigma=C D$ for a free body corresponds to the length of a holder $l=O D$ of a fixed pendulum.

3. The first integrals of the system (69), (70) can be automatically obtained through the Eqs. (50), (67), (68) after substitutions (72) (see also [8, 9]):

$$
\begin{gathered}
\Theta_{1}^{\prime}\left(w_{2}, w_{1} ; \alpha\right)= \\
=\frac{w_{2}^{2}+w_{1}^{2}-b w_{2} \sin \alpha+\sin ^{2} \alpha}{w_{1} \sin \alpha}= \\
=C_{1}=\text { const }
\end{gathered}
$$

$$
\begin{gathered}
\Theta_{2}^{\prime}\left(w_{2}, w_{1} ; \alpha\right)= \\
=G\left(\sin \alpha, \frac{w_{2}}{\sin \alpha}, \frac{w_{1}}{\sin \alpha}\right)= \\
=C_{2}=\text { const. } \\
\Theta_{3}^{\prime}\left(w_{2}, w_{1} ; \alpha, \beta_{1}\right)= \\
=-\beta_{1} \pm \frac{1}{2} \operatorname{arctg} \frac{w_{1}^{2}-w_{2}^{2}+b w_{2} \sin \alpha-\sin ^{2} \alpha}{w_{1}\left(2 w_{2}-b \sin \alpha\right)}= \\
=C_{3}=\text { const. }
\end{gathered}
$$


The second group of analogies deals with the case of a motion with the constant velocity of the center of mass of a body, i.e., when the property (28) holds. In this case the dynamical part of the motion equations under certain conditions is reduced to a system (32)(36).

Then, under conditions (28), (37), (38), (71) $\left(w_{k} \leftrightarrow Z_{k}\right)$ the reduced dynamical part of the motion equations (system (33)-(36)) has the form of analytical system

$$
\begin{gathered}
\alpha^{\prime}=-Z_{2}+b\left(Z_{1}^{2}+Z_{2}^{2}\right) \sin \alpha+b \sin \alpha \cos ^{2} \alpha, \\
Z_{2}^{\prime}=\sin \alpha \cos \alpha-Z_{1}^{2} \frac{\cos \alpha}{\sin \alpha}+b Z_{2}\left(Z_{1}^{2}+Z_{2}^{2}\right) \cos \alpha- \\
-b Z_{2} \sin ^{2} \alpha \cos \alpha, \\
Z_{1}^{\prime}=Z_{1} Z_{2} \frac{\cos \alpha}{\sin \alpha}+b Z_{1}\left(Z_{1}^{2}+Z_{2}^{2}\right) \cos \alpha- \\
-b Z_{1} \sin ^{2} \alpha \cos \alpha, \\
\beta_{1}^{\prime}=Z_{1} \frac{\cos \alpha}{\sin \alpha},
\end{gathered}
$$

in this case, we choose the constant $n_{1}$ as follows: $n_{1}=n_{0}$.

If the problem on the firs integrals of the system (69), (70) is solved using Corollary 6, the same problem for the system (76), (77) can be solved by the following Theorem 7.

At the beginning, we note that one of the firs integrals of the system (76), (77) has the following form (see [10]):

$$
\begin{gathered}
\Theta_{1}^{\prime \prime}\left(Z_{2}, Z_{1} ; \alpha\right)= \\
=\frac{Z_{2}^{2}+Z_{1}^{2}-b Z_{2} \sin \alpha+\sin ^{2} \alpha}{Z_{1} \sin \alpha}= \\
=C_{1}=\text { const. }
\end{gathered}
$$

Later on, we study an additional firs integral of the third-order system (76) using, in this case, the firs integral (78). For this we introduce the following notations and new variables (comp. with $[11,12])$ :

$$
\tau=\sin \alpha, Z_{k}=u_{k} \tau, k=1,2, p=\frac{1}{\tau^{2}} .
$$

Then the problem on explicit form of the desired firs integral reduces to solving of the linear inhomogeneous equation:

$$
\begin{gathered}
\frac{d p}{d u_{2}}=\frac{2\left(u_{2}-b\right) p+2 b\left(1-U_{1}^{2}\left(C_{1}, u_{2}\right)-u_{2}^{2}\right)}{1-b u_{2}+u_{2}^{2}-U_{1}^{2}\left(C_{1}, u_{2}\right)}, \\
U_{1}\left(C_{1}, u_{2}\right)=\frac{1}{2}\left\{C_{1} \pm \sqrt{C_{1}^{2}-4\left(u_{2}^{2}-b u_{2}+1\right)}\right\},
\end{gathered}
$$

in this case, an additive constant $C_{1}$ can be chosen as follows: $b^{2}+C_{1}^{2}-4 \geq 0$.
The last fact means that we can fin another transcendental firs integral in the explicit form (i.e., as a finit combination of quadratures). Here, the general solution of Eq. (80) depends on an arbitrary constant $\mathrm{C}_{2}$. We omit the calculation, but note that the general solution of the linear homogeneous equation obtained from (80) even in the particular case $b=C_{1}=2$ has the following solution:

$$
\begin{gathered}
p=p_{0}\left(u_{2}\right)=C\left[\sqrt{1-\left(u_{2}-1\right)^{2}} \pm 1\right] \times \\
\times \exp \left[\sqrt{\left.\frac{1 \mp \sqrt{1-\left(u_{2}-1\right)^{2}}}{1 \pm \sqrt{1-\left(u_{2}-1\right)^{2}}}\right], C=\text { const. }}\right.
\end{gathered}
$$

Then the desired additional firs integral has the following structural form (which is similar to the transcendental firs integral from the plane-parallel dynamics):

$$
\begin{gathered}
\Theta_{2}^{\prime \prime}\left(Z_{2}, Z_{1} ; \alpha\right)= \\
=G\left(\sin \alpha, \frac{Z_{2}}{\sin \alpha}, \frac{Z_{1}}{\sin \alpha}\right)=C_{2}=\text { const }
\end{gathered}
$$

in this case, we use the notations and substitutions (79).

Thus, for the integration of the fourth-order system (76), (77), we have found two independent firs integrals. For the complete its integration, it suffice to fin one (additional) firs integral that "attaches" Eq. (77).

The desired firs integral can be obtained by the following relation:

$$
\sin \left[2\left(\beta_{1}+C_{3}\right)\right]= \pm \frac{2 Z_{1}-C_{1} \sin \alpha}{\sqrt{b^{2}+C_{1}^{2}-4} \sin \alpha} .
$$

In principle, in order to obtain an additional invariant relation that "attaches" Eq. (77), we could stop on the last equation. In this case, we must formally substitute the left-hand side of the firs integral (78) into the last expression instead of $C_{1}$.

But we perform some transformations which allow to obtain the following fina explicit form of the additional firs integral:

$$
\begin{gathered}
\Theta_{3}^{\prime \prime}\left(Z_{2}, Z_{1} ; \alpha, \beta_{1}\right)= \\
=-\beta_{1} \pm \frac{1}{2} \operatorname{arctg} \frac{Z_{1}^{2}-Z_{2}^{2}+b Z_{2} \sin \alpha-\sin ^{2} \alpha}{Z_{1}\left(2 Z_{2}-b \sin \alpha\right)}= \\
=C_{3}=\text { const. }
\end{gathered}
$$

Theorem 7 Three first integrals (78), (81), (82) of the system (76), (77) are the transcendental functions of its own phase variables and are expressed as a finite combination of elementary functions. 
Theorem 8 Three first integrals (78), (81), (82) of the system (76), (77) are equivalent to three first integrals (73), (74), (75) of the system (69), (70).

Indeed, the couples of the firs integrals (78), (73) and (82), (75) coincides, if we substitute $b=-b_{*}$. And finall, we need to identify the phase variables $Z_{k}, k=1,2$, for the system (76), (77) with the phase variables $w_{k}, k=1,2$, of the system (69), (70). Because of their cumbersome character, the similar arguments concerning of the couples of the firs integrals (81), (74), we do not represent.

Thus, we have the following topological and mechanical analogies in the sense explained above.

(1) A motion of a fi ed physical pendulum on a spherical hinge in a fl wing medium (nonconservative force fields)

(2) A spatial free motion of a rigid body in a nonconservative force fiel under a tracing force (in the presence of a nonintegrable constraint).

(3) A spatial composite motion of a rigid body rotating about its center of mass, which moves rectilinearly and uniformly, in a nonconservative force field

On more general topological analogues, see also $[8,9,12,13,15,16,17]$.

Acknowledgements: This work was supported by the Russian Foundation for Basic Research, project no. 19-01-00016.

\section{References:}

[1] Shamolin M. V. Some questions of the qualitative theory of ordinary differential equations and dynamics of a rigid body interacting with a medium, J. Math. Sci., 2002, 110, no. 2, pp. 2526-2555.

[2] Shamolin M. V. New integrable cases and families of portraits in the plane and spatial dynamics of a rigid body interacting with a medium, J. Math. Sci., 2003, 114, no. 1, pp. 919-975.

[3] Shamolin M. V. Foundations of differential and topological diagnostics, J. Math. Sci., 2003, 114, no. 1, pp. 976-1024.

[4] Shamolin M. V. Classes of variable dissipation systems with nonzero mean in the dynamics of a rigid body, J. Math. Sci., 2004, 122, no. 1, pp. 2841-2915.

[5] Shamolin M. V. On integrability in elementary functions of certain classes of nonconservative dynamical systems, J. Math. Sci., 2009, 161, no. 5, pp. 734-778.
[6] Shamolin M. V. Dynamical systems with variable dissipation: approaches, methods, and applications, J. Math. Sci., 2009, 162, no. 6, pp. 741-908.

[7] Shamolin M. V. Classificatio of complete integrability cases in four-dimensional symmetric rigid-body dynamics in a nonconservative field J. Math. Sci., 2010, 165, no. 6, pp. 743754.

[8] Trofim v V. V., and Shamolin M. V. Geometric and dynamical invariants of integrable Hamiltonian and dissipative systems, J. Math. Sci., 2012, 180, no. 4, pp. 365-530.

[9] Shamolin M. V. Comparison of complete integrability cases in Dynamics of a two-, three-, and four-dimensional rigid body in a nonconservative field J. Math. Sci., 2012, 187, no. 3, pp. 346-459.

[10] Shamolin M. V. Some questions of qualitative theory in dynamics of systems with the variable dissipation, J. Math. Sci., 2013, 189, no. 2, pp. 314-323.

[11] Shamolin M. V. Variety of Integrable Cases in Dynamics of Low- and Multi-Dimensional Rigid Bodies in Nonconservative Force Fields, J. Math. Sci., 2015, 204, no. 4, pp. 479-530.

[12] Shamolin M. V. Classificatio of Integrable Cases in the Dynamics of a Four-Dimensional Rigid Body in a Nonconservative Field in the Presence of a Tracking Force, J. Math. Sci., 2015, 204, no. 6, pp. 808-870.

[13] Shamolin M. V. Some Classes of Integrable Problems in Spatial Dynamics of a Rigid Body in a Nonconservative Force Field, J. Math. Sci., 2015, 210, no. 3, pp. 292-330.

[14] Shamolin M. V. New Cases of Integrable Odd-Order Systems with Dissipation, Doklady Mathematics, 2020, 101, no. 2, pp. 158-164.

[15] Shamolin M. V. New Cases of Integrable Odd-Order Systems with Dissipation, Doklady Mathematics, 2020, 101, no. 2, pp. 158-164.

[16] Shamolin M. V. New Cases of Homogeneous Integrable Systems with Dissipation on Tangent Bundles of Four-Dimensional Manifolds, Doklady Mathematics, 2021, 103, no. 2, pp. 85-91.

[17] Shamolin M. V. First Integrals of Systems with Three Degrees of Freedom and Dissipation, 2018 International Conference Stability and Oscillations of Nonlinear Control Systems (Pyatnitskiys Conference) (Moscow, 30 May - 1 June 2018), IEEE, 2018, pp. 1-4.

Creative Commons Attribution License 4.0 (Attribution 4.0 International, CC BY 4.0)

This article is published under the terms of the Creative Commons Attribution License 4.0

https://creativecommons.org/licenses/by/4.0/deed.en_US 